\title{
Personalized Educational Paths through Self-Modifying Learning Objects
}

\author{
George Pashev, George Totkov, Hristina Kostadinova, Hristo Indzhov
}

\begin{abstract}
The paper presents a formal model for generation of personalized learning paths. The paths consist of self-modifying learning activities suitable for the achievement of course goals. The course goals (as defined by the teacher) are a list of functions/predicates with specific slots and include obligatory activities required for the automatized construction of learning paths. Further, the problems related to automatized learning path construction are identified and solved with the use of an original approach. The approach includes (but is not limited to): introduction of different points of view (aspects) for learning objects; automatized gathering/accumulation of metadata for learning activities; generation of personalized learning paths using set of student achievements, etc.

Key words: adaptive e-learning, generation of e-learning paths, self-modifying learning objects, automatized metadata gathering, revised Bloom taxonomy
\end{abstract}

\section{INTRODUCTION}

In the e-learning context, the level of personalization of the learning experience is the measurement for quality and effectiveness of education. Said differently, the learning paths of students may and will differ from one another based on their skills/knowledge, learning goals, motivation and individual learning styles. According to her/his personal traits the learner may take one course or another or even different learning paths within a single course. If personalized the learning content could be better understood by the learner and it could be more relevant and acceptable by her/him [15, 5, 16, 14].

A detailed discussion of personalization and adaptation of e-learning processes and suitable tools for authoring of used learning objects (LOs) is presented in [14, 15]. It is also notable that the support for personalization and adaptation by the current tools of the trade is not on the desired level. It boils down to the way LOs are combined together to build a learning path. Sequencing of learning materials and learning activities (LAs) is often very simplistic and not a subject to advanced strategies.

In systems such as AHA! [5] and ELM-ART [16] the sequence of LOs is developed and delivered step by step through link-annotation [4]. In [2, 10] for example, the sequence is defined initially, and maintained on occasion [13]. Other systems like Lecomps [13] use advanced techniques (constraint logic based engine) to perform the course construction and sequencing.

The systems under review, however, do not provide a solution to basic problems related to learning path construction. The problems can quickly be identified by answering the fundamental questions below:

Q1: How different LOs can be evaluated from different points of view (aspects)?

Q2: How collection/accumulation of metadata for LAs (in our particular case selfmodifying LAs) can become part of the e-learning process?

Q3: How the process of student assessment in the context of a specific LA (with dynamically generated metadata) can be automated?

Q4: How obligatory LA can be included in personalised e-learning path (eLP)?

This paper answers the above raised questions and creates the foundation of a

Permission to make digital or hard copies of all or part of this work for personal or classroom use is granted without fee provided that copies are not made or distributed for profit or commercial advantage and that copies bear this notice and the full citation on the first page. Copyrights for components of this work owned by others than ACM must be honored. Abstracting with credit is permitted. To copy otherwise, or republish, to post on servers or to redistribute to lists, requires prior specific permission and/or a fee. Request permissions from Permissions@acm.org.

CompSysTech'16, 23-24 June 2016, Palermo, Italy

Copyright (C) 2016 ACM. ISBN 978-1-4503-4182-0/16/06...\$15.00

http://dx.doi.org/10.1145/2983468.2983516 
software system for generation of personalized learning paths. A short review of existing solutions/approaches follows.

A possible answer to Q2 is presented in [7]. The main pillar of the presented framework is a two-dimensional table (RBTt) with 6 rows and 4 columns and is based on the Revised Bloom's Taxonomy [1, 3]. It consists of more than 450 test items that can be used efficiently for construction of digital activities' description and form large variety of learning paths in different cognitive levels. These activities can then be used to dynamically propose the most appropriate learning object, according to the learners' needs.

[4] presents a framework for gathering/accumulating of metadata according to LOM standard in the case of test items. There is a drawback in the described approach with regard to the limited number of test items used for metadata generation. The above mentioned disadvantage can be overcome by transforming the table with the free answer questions and expand it in order to cover all the needed metadata fields. The proposed improved method is based on the following fundamental ideas: first, metadata is going to be collected by providing a dialog for learners (or experts in the subject domain) after they are already familiar with the LA (read the LA if it is in a text format) and second, the accumulated answers will be graded (by the above mentioned learners/experts) and used as metadata for the LA. This can and will lead to an optimized process for gathering of the required information for the learning object's description.

With regard to Q4: Formal models like PeU [14] define the e-course statically by a graph structure, called „course model“. Adaptive systems like [13] don't allow inclusion of obligatory LA in the course goals (CGs).

The paper proposes answers the above mentioned questions introducing a suitable formal model. A software system PUAdapt has been created to experiment with the models.

\section{Our Approach}

Learning object is very common term in an e-learning literature. Referring to The Learning Technology Standards Committee learning objects are defined as "any entity, digital or non-digital, which can be used, re-used or referenced during technology supported learning". Considering the LO idea, learning process may be divided into smaller, reusable learning units. According to [8], flexibility is an ability of a process to execute on the basis of a partially defined model where the full specification is made at runtime and may be unique to each instance [8]. We are going to present a slightly modified version of the presented in [16] definition of flexibility. Flexibility is an ability of a learning process to execute on the basis of a partially defined model and self-modified LOs where the full specification and used LOs are made at runtime and may be unique to each instance.

Adaptability in learning management systems is defined as a potential to deliver personalized LAs to each student. In our model, content, description and order of these LAs are variables and depend on learning goals, student's knowledge and preferences, accumulated experience, previous successful learning paths [9], etc. The teacher defines the course goals as list of functions/predicates with specific number of slots and could point concrete learning activities to be included in the automatized constructed learning paths.

\section{Answer to Q1: Aspects of the LOs}

An aspect model of the learning object $L$ is presented by

$L=\left(I_{L}, N_{L}, D_{L}, P_{L}, D_{M L}, V_{L}\right)$,

where:

- $I_{L}: L \rightarrow Z ; z \in \mathbb{N}$ is an aspect identifier;

- $N_{L}$ is a name of an L;

- $D_{L}$ is a distance function $D_{L}: P_{L_{x_{1}}} \times P_{L_{x_{2}}} \rightarrow a_{1} ; a_{1} \in \mathbb{R}$;

- $x_{1} \neq x_{2} ; x_{1}, x_{2}=\overline{1,\left|P_{L}\right|} ; x_{1}, x_{2} \in \mathbb{N}$; 
- $\quad P_{L}$ is a set of points in $L: P_{L}=\left\{P_{L_{n}}: n=\overline{1, t} ; n, t \in \mathbb{N}\right\}$;

- $D_{M_{L}}$ is a set of dimensions, where $d_{\mu} \in D_{M_{L}}$;

- $D_{M_{L}}=\left\{d_{\mu}: \mu=\overline{1, b_{4}} ; \mu, b_{4} \in \mathbb{N}\right\}$;

- $V_{L}$ is a function: $V_{L}: P_{L_{j}} x d_{k} \rightarrow b_{5} ; b_{5} \in \mathbb{R} ; j=\overline{1, t} ; k=\overline{1, b_{4}}$.

The dimensions correspond to the RBTt [3] and are not limited to them only. All of them can be represented as functions with specific input and output parameters. Most of those functions are predicates as they return Boolean values. An example of a non-predicate function is seen (concept, [aspects], resource function name) which returns a real number.

In the following notation a list relation notation is used, which is formally defined as $\left[S_{\chi}\right]: S_{\chi} \rightarrow\left(S_{1}, \ldots S_{\varsigma}: \chi \in \overline{1, \varsigma} ; \chi, \varsigma \in \mathbb{N}\right)$.

\section{Answer to Q2: Generation of metadata for LOs}

The metadata generation function is defined formally as:

$F:$ Level $x \Omega \longrightarrow[(\omega,[$ slot $]$, val $)]$

where $\Omega \in\{$ Facts,Concepts, Procedures, Meta - knowledge $\}$ is an activity in RBTt; Level is a cognition level in RBTt; $\omega \in$ Level $x \Omega$ is a verb in RBTt; $[(\omega,[$ slot $]$, val $)]$ is a list of triplets (action verb; list of slots, which is different for each verb in RBTt; returned value).

The content model which consists of the LAs offered to the learners is a significant part of each adaptive e-learning system [12]. The quality of these objects is significant for the final result and can reflect to the satisfaction of the leaners needs. In order to achieve a real personalized learning process, the e-learning system is supposed to follow different paths in the content model, depending the learners needs (preferences and level of knowledge) [12]. An algorithm, based on the teaching strategy has to choose the most appropriate LAs for the concrete user. This is the reason why each LA has to be described with an appropriate set of information about itself, i.e. the so called metadata. Collecting metadata for each LA used in the e-course is long and time consuming process. In what follows we present an approach for generation of set of metadata as answers to questions about content of corresponding LA. The basic difficulty is to propose the most appropriate questions in order to accumulate answers of students (or experts in the subject area) as possible descriptions of given LA.

The dialog for metadata collection is going to be started after given LA is finished by a group of learners. The system proposes to each learner a list of fixed number of action verbs according to the Revised Bloom's Taxonomy. The verbs are ordered by knowledge dimension (facts, concepts, procedures, meta-knowledge) and continuously in different cognitive process dimensions (remembering, understanding, applying, analysing, evaluating and creating). Each action verb is related with one or more didactical element (facts, concepts, examples, procedures, etc.). These didactical elements vary depending on the subject domain and their list can be enlarged. The complete list of the action verbs consists of dozens of verbs, which are going to be used in the process of generating LAs' description. The method which is going to be used consists of several steps:

Step 1. Learner has to choose if the proposed actions verbs are relevant to the LA by checking them. A list with action verbs is provided by the system and the question which the learner has to answer is: "Please, choose one or more action verbs, relevant to the learning material!"

Step 2. Learner has to name the concepts, facts, procedures and other didactical elements which are related with checked action verbs which are used in the LA. The question which learner has to answer is: "Please, fill the list of the didactic elements (concepts, facts, procedures etc.), which are related with the checked action verb!"

Step 3. The data collected by the learners are stored and graded further by the teacher 
or an expert in the subject domain.

Step 4. Statistics of the most commonly checked action verbs is provided as well the list of the corresponding didactical elements, too.

The description of the LA is generated by sorting the collected data by the number of the occurrences in the leaners answers. The most commonly checked verbs are with precedence. Additional analysis of the accumulated metadata can lead to different order of the list with the basic concepts, facts, procedures and other didactical elements that are included in the learning material.

Thus, LOs used in e-learning process are self-modifying objects. A list of modifications generates new sets of Los metadata.

Answer to Q3 \& Q4: Automatized learner assessments and incorporation of obligatory LAs in personalized learning paths

Fig. 1. presents the student e-learning work-flow. The student can choose among a list of adaptive courses she/he is enrolled in.

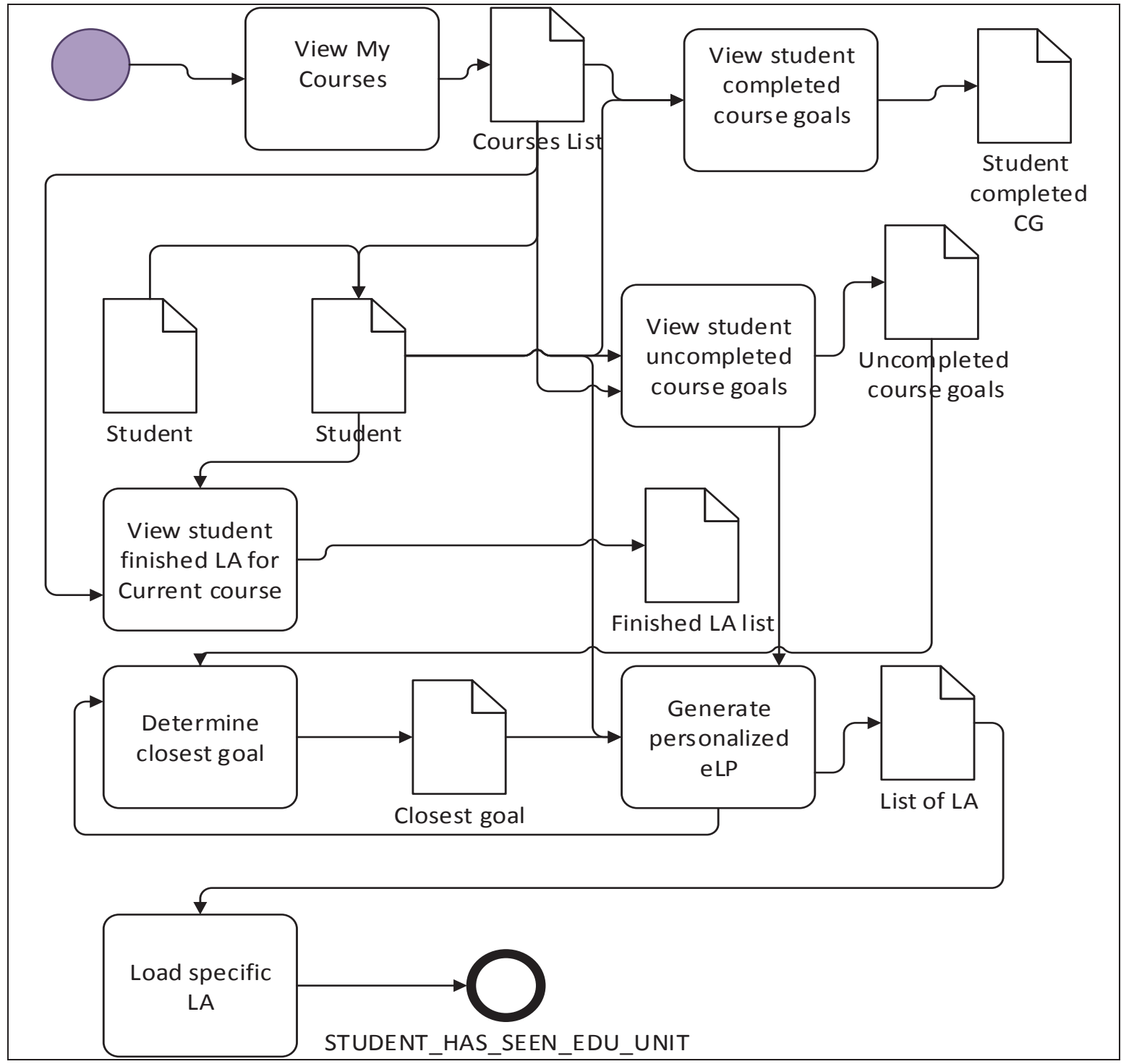

Figure 1. The Student e-Learning Work-flow

Then, $\mathrm{S}$ is able to view her/his uncompleted goals in the course, ordered by the closest locality to its current state (a point of $S$ in Multidimensional Space - MDS [11]). The closest goal to the radius-vector of $S$ (current state of $S$ ) in MDS is then chosen and list of LAs is generated by the algorithm for generation of personalised eLP (see fig. 3.).

Upon a display event of a specific LA to a student, a Student Examination Automation 
Sub-process is executed. The available metadata in the form, returned by $F$ is exported as a list. If the metadata already gathered for the specific LA are enough a static test is generated. The test is then displayed to $\mathrm{S}$ to submit a solution. After $\mathrm{S}$ submits a solution, the grade is automatically generated, since the right answers are available in the metadata used for the generation of the test. If there is not enough metadata, an accumulative test for metadata generation is displayed to $\mathrm{S}$. The solution given to the accumulative test is then sent to the teacher to get a grade.

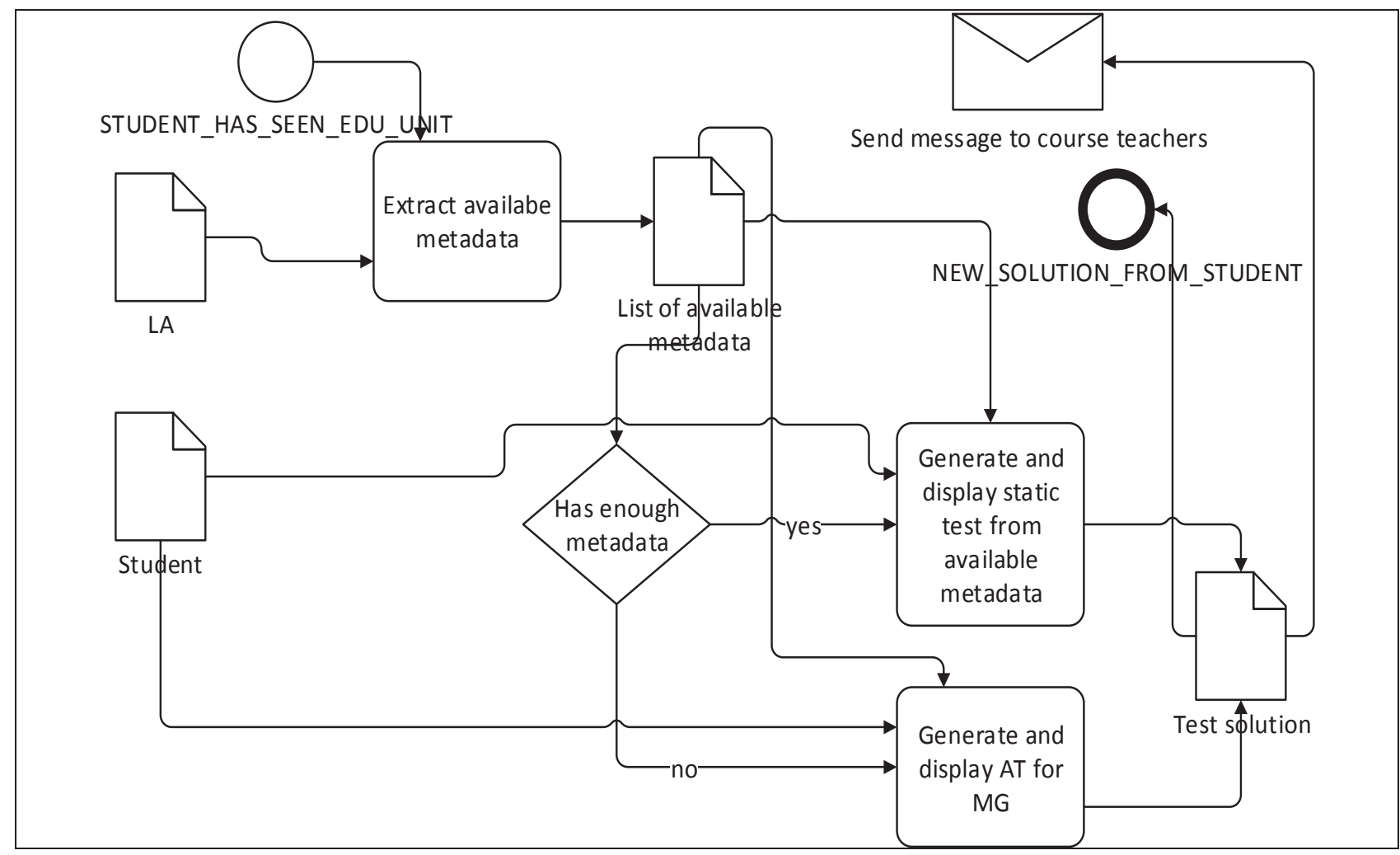

Figure 2. Student Examination Automation Sub-process

Examples of predicates as possible elements, returned by $F$ are:

- $\quad($ pred, [concept 1 , concept 2 ], true) - denotes that concept 1 is met before concept 2

- (initial, [concept ${ }_{1}$ ], true) - denotes that concept ${ }_{1}$ is an initial concept;

- (obligLA, [LA $\mathrm{LA}_{1}$, true) - denotes that $\mathrm{LA}_{1}$ is obligatory for a specific $C G$

The predicate obligLA is used to make a $L A_{1}$ obligatory for selection by the $e L P$ Generation Algorithm.

Algorithm for Personalised eLP Generation

The algorithm for eLP generation ( $\left.e L P_{-} G A\right)$ can be formally defined as a function:

$e L \mathrm{P}_{\mathrm{GA}}:$ Student $\rightarrow[(\zeta, \varsigma, v)]$

The input of the function is a student object (SO). The function returns a list of triplets $[(\zeta, \zeta, v)]$, which correspond to LAs containing: $\zeta$ - a display URL of the current $L A$, $\varsigma$ - a distance length from LA to current SO in MDS and $v$ - short name of the LA.

In what follows let us denote:

- $R V e c(O b j)$ - representation of Obj as a radius-vector in MDS;

- $\quad V_{L}(R V e c(O b j),(\omega,[s l o t]))$ - the returned value for a given $R V e c(O b j)$ for a dimension, which is searched by an action verb and a list of slots;

- search: BasePoint $x$ reachRadius $x$ searchFilter $x n \rightarrow[[(\omega,[$ slot $], v a l)]]-$ the function which finds $n$ closest points to BasePoint, accessible within the reachRadius and comply with the dimension predicate 
searchFilter: booleanExpression $\rightarrow \rho: \rho \subseteq P_{L}$;

- searchGE: BasePoint $x$ reachRadius $x$ searchFilter $x n \rightarrow[[(\omega,[$ slot $]$, val $)]]-$ the function which (in contrast of search) returns a list of points which have absolute values greater or equal than the absolute value of BasePoint. The list has maximal size of $n$ elements, which comply with the searchFilter;

- orderTo: BasePoint $x[[(\omega,[$ slot $], v a l)]] \rightarrow[[(\omega,[$ slot $], v a l)]]$ - orders a list of points in MDS in the closest order to BasePoint.

- isGE(RVec(objWhat), RVec(objToWhat)) - a Boolean function which checks whether the absolute value of $R V e c(o b j W h a t)$ is greater than $R V e c$ (objToWhat) and if it is returns true.

Fig. 3. presents the eLP_GA.

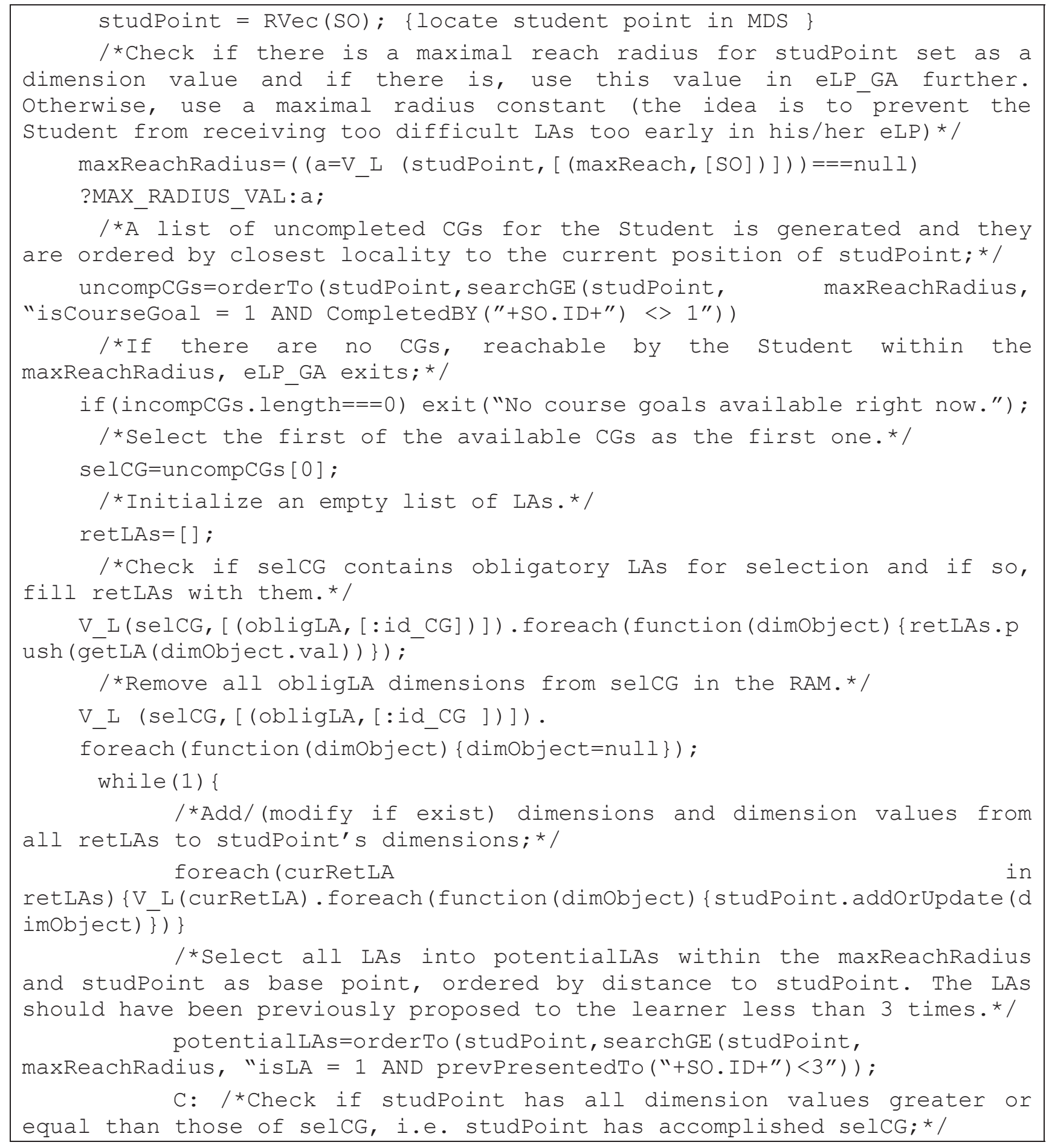




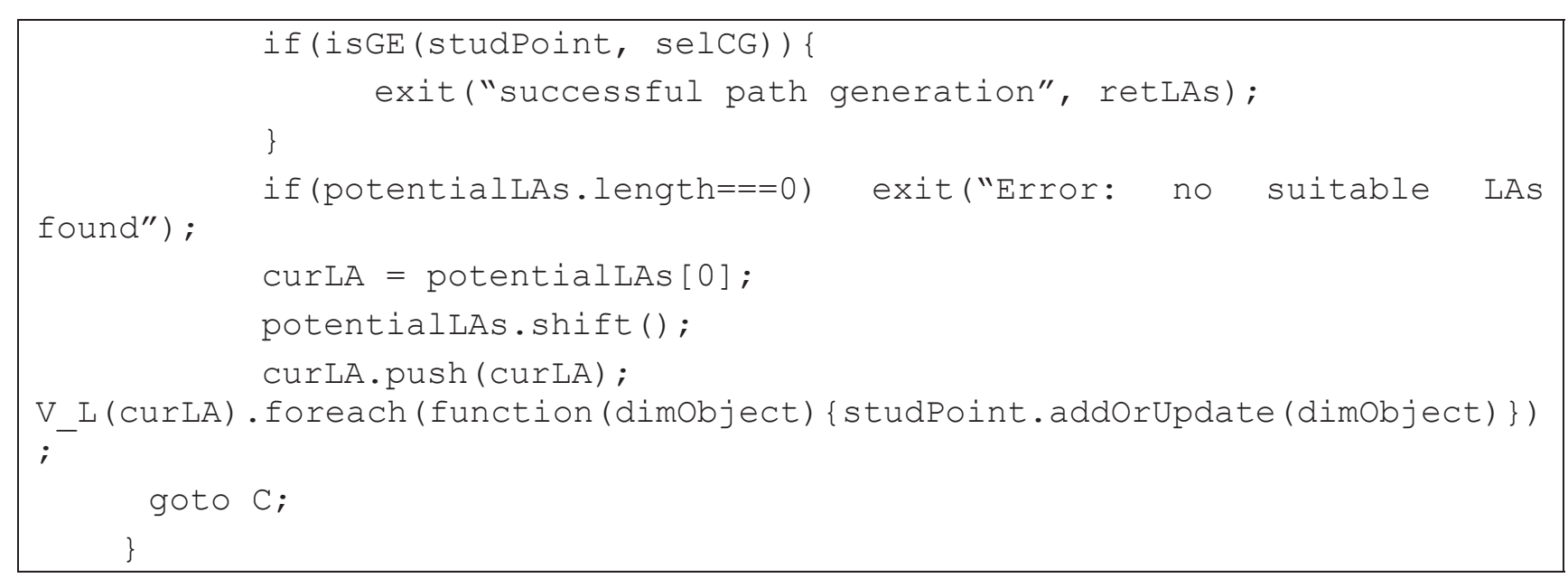

Figure 3. Algorithm for Personalized eLP Generation

\section{Conclusion and future work}

The presented conceptual framework can lead to an automatized metadata generation and can provide additional advantages to the adaptive e-learning process. One such advantage is to find out the profile of missing LAs needed for a successful CG completion by students. Furthermore, the process of metadata accumulation for LAs can become faster by utilizing student answers to questions like: "Which of the following course goals is most relevant to the current LA?". If the majority of students choose a specific CG most frequently, its metadata can be transferred to that specific LA. Students themselves can be given the option to define their personal CGs in order to make their educational process more personalized. CGs can also be assigned to specific student groups. Mandatory CGs can be included in adaptive courses to give the students the opportunity to profile their knowledge in a desired direction.

\section{REFERENCES}

[1] Anderson, L., Krathwohl, D. R. (Eds.). A taxonomy for learning, teaching and assessing: A revision of Bloom's Taxonomy of educa-tional objectives: Complete edition, New York Longman, 2001.

[2] Baldoni, M., Baroglio, C., Brunkhorst, I., Marengo, E., and Patti, V. "ReasoningBased Curriculum Sequencing and Validation: Integration in a Service-Oriented Architecture" In EC-TEL 2007, LNCS 4753, pp. 426-431.

[3] Bloom, B. Taxonomy of Educational Objectives. Published by Allyn and Bacon, Boston, MA Copyright (c) 1984 by Pearson Education (1956).

[4] Brusilowsky, P. Adaptive Hypermedia" Journal of User Modeling and User-Adapted Interaction, Vol. 11, 2001.pp. 87-110.

[5] De Bra, P., Smits, D., and Stash, N. "Creating and delivering adaptive courses with AHA!" In EC-TEL 2006, pp. 21-33.

[6] Kostadinova, Hr., Totkov, G., Blagoev, D. Automatized Generation of Metadata for Learning Objects, Proceedings of the 4-th National Conference "Learning in Information Society“, 26-27 May 2011, Plovdiv, pp. 44-52.

[7] Kostadinova, Hr., Totkov, G., Indzhov, Hr. Adaptive E-learning System Based on Accumulative Digital Activities in Revised Bloom's Taxonomy, CompSysTech'12, 22-23 June 2012, Ruse, Bulgaria, pp. 368-375.

[8] Mangan, P. and Sadiq, S. On building workflow models for flexible processes. In Proceedings of the 13th Australasian database conference - Volume 5 (ADC '02). Australian Computer Society, Inc., Darlinghurst, Australia, 2002. pp. 103-109.

[9] Pashev, G; Totkov, G; Dynamic Determination of Personalized Educational Paths; Proceedings of the National Conference on "Education and Research in the Information 
Society", Plovdiv, May, 2014; Institute of Mathematics and Informatics Bulgarian Academy of Sciences, Association for the Development of the Information Society, pp. 161-170 (in Bulgarian).

[10] Sangineto, E., Capuano, N., Gaeta, and M., Micarelli, A. Adaptive course generation through learning styles representation, Universal Access in the Information Society (UAIS), Vol. 7(1-2), 2008, pp. 1-23.

[11] Shamos, M. I. and Hoey, D. J. "Closest-Point Problems." Proe. 16th Annual Symposium on Foundations of Computer Science. October, 1975.

[12] Shute, V., B. Towle, Adaptive E-learning. Educational Psychologist Volume: 38, Issue: 2, Publisher: Lawrence Erlbaum Associates, Inc. 10, 2003, pp. 105-114.

[13] Sterbini A, and Marco Temperini Adaptive Construction and Delivery of WebBased Learning Paths, 39 ${ }^{\text {th }}$ ASEE/IEEE Frontiers in Education Conference, October 18-21 2009, San Antonio, TX, W1C1-6.

[14] Totkov G., Somova, E., Sokolova, M. Modelling of e-Learning Processes: an Approach Used in Plovdiv e-University, Int. Conf. on Computer Systems and Technologies (e-learning), Comp-SysTech'04, 17-18 June 2004, Rousse, IV.12.1 - IV.12.6.

[15] Totkov, et al; PUAdapt Module, Modern tendencies in e-learning, ISBN 978-9548852-46-3; Plovdiv, 2014, pp. 162-173 (in Bulgarian).

[16] Weber G., and Brusilovsky P. Elm-art: An adaptive versatile system for web-based instruction." Int. Journal of Al in Education, Vol.12(4), 2001, pp. 351-384.

\section{About the authors}

George Pashev, M.Eng, PhD Student, University of Plovdiv „Paisii Hilendarski“, tel:00359895662140, georgepashev@uni-plovdiv.bg

George Totkov, Prof. DSc. University of Plovdiv „Paisii Hilendarski“ tel: 0035932261240; totkov@uni-plovdiv.bg

Hristina Kostadinova, PhD, Chief Assistant, New Bulgarian University, hkostadinova@gmail.com

Hristo Indzhov, PhD, „Pastel Studios“ Ltd., h.indzhov@pastelstudios.com 